\title{
A Hybrid of BFO and MCS Algorithm for Channel Estimation of MIMO-OFDM System
}

\author{
Dr.R.Manjith \\ Associate Professor, Dept of ECE, Dr.Sivanthi Aditanar College of Engineering, Tiruchendur, Tamilnadu, India
}

\begin{abstract}
In this paper, challenges regarding the provision of channel state information (CSI) in multiple input multiple output orthogonal frequency division multiplexing (MIMO-OFDM)) systems are addressed. A novel scheme that utilizes a Hybrid of Bacterial Foraging and Modified Cuckoo Search (HBFOMCS) optimization together to design pilot symbols that minimizes the channel estimate mean squared error (MSE) is proposed. The optimal selection of pilot subcarriers is a combinatorial problem that requires heavy computations. To reduce the computational complexity, the HBFOMCS optimization is utilized to determine the position of pilot subcarriers. Then, for a given pilot placement obtained by the HBFOMCS algorithm, the MSE of least squares (LS) and Minimum Mean Square Error(MMSE) based channel estimation are computed. Simulation results indicate that, the proposed pilot symbol design provides better channel estimate MSE as well as the bit error rate (BER) performance with reduced computational complexity for MMSE method when compared with the LS method and other conventional optimization based pilot design.
\end{abstract}

Keywords: HBFOMCS, LS channel estimation, MMSE channel estimation, MIMO-OFDM,MSE.

\section{INTRODUCTION}

The telecommunication services demand high data rates with reliability. However, to achieve high data rates, it is necessary to use a wide spectral bandwidth, which makes the system economically unfeasible. Another problem is that, in this scenario, the channel becomes very selective [1], impairing the reliability of the received information. In order to minimize these problems, digital signal processing techniques combined with designing transceivers strategies are used, where Multiple Inputs and Multiple Outputs (MIMO) deserves mention. MIMO systems use multiple antennas to transmit and to receive signals [2]. The multiple signals transmitted and the multiples replicas obtained in the receiver can be combined to increase the robustness (diversity) or the data rate (multiplexing). Orthogonal Frequency Division Multiplexing technique (OFDM) [3] are commonly used to overcome the ISI (Inter Symbol Interference) introduced by multipath channel. This technique is employed in several digital communications standards, such as DVB-T, DVB-T2, Wi-Fi, Wi-Max, IEEE 802.11 and others. Therefore, future telecommunication systems tend to combine both techniques mentioned above known as MIMO-OFDM systems [4].Depending on the designed scheme, a system operating with MIMO-OFDM can provide robustness against frequency selective and timevariant channels, and/or to obtain multiplexing gain.

In a fading channel environment, channel estimation is an essential part for decoding the received signals. For a MIMO-OFDM system where two or more transmitting antennas are used, this channel estimation becomes a challenging task. The major challenge in this scenario is to obtain accurate channel estimation for detection of the information symbols, since the receiver requires the Channel State Information (CSI) to equalize the received symbols, due to the phase rotation and amplitude attenuation caused by the channel. However, in these systems, knowledge of the CSI at the receiver is necessary for interference cancellation, coherent detection and demodulation. The CSI can be obtained through blind, semi blind and training based channel estimation methods [5]. The blind channel estimation is carried out by evaluating the statistical information of the channel and certain properties of the transmitted signals [6]. Semi blind channel technique is hybrid of blind and training technique, utilizing pilots and other natural constraints to perform channel estimation. To this end, channel estimation methods based on pilot symbols can be applied to obtain CSI in MIMO-OFDM systems[7] 2009) and [8].

In order to detect and recover the transmitted data optimally, the CSI needs to be estimated. In pilot symbol method pilot tones are multiplexed with the data stream and they are only used for channel estimation purpose. Hence in practice there is a trade-off between the density of the pilots and the estimation quality. The CSI is estimated based on utilizing the initial channel state on pilot position by dividing the received pilots with the known transmitted pilots. The Least Square (LS) and Minimum Mean Square Error (MMSE) techniques are widely used for channel estimation when training symbols are available [9]. In LS, the estimation process is simple but the problem is that it has high mean square error .Due to its simplicity; however, the LS method has been widely used for channel estimation. However, in Low SNR, the MMSE method is better than that of LS method, but its main problem is its high computational complexity. The pilot symbol based MMSE channel estimation method provides better performance of estimating the channel information accurately at low SNR values.

In pilot tones based LS and MMSE channel estimation methods, pilot tones are inserted into each subcarrier at one OFDM symbol or inserted to the subset of subcarriers with a specific period [9]. However, the design of the location of pilot tones will directly affect the performance 
of the channel estimation algorithms. The optimization of the pilot tones for MIMO-OFDM systems has been investigated recently. This gives us a strong drive to further exploit the channel estimation method that gives the best performance. In [10], a scheme for designing pilot symbols for OFDM-based CR systems is proposed. In [11], [12], and [13] pilot tones optimization using ABC, $\mathrm{DE}$ and PSO algorithms for LS channel estimation method are proposed.

In this paper, a novel method that utilizes the hybrid of bacterial foraging and modified cuckoo search (HBFOMCS) optimization together is proposed to design pilot tones for LS and MMSE channel estimation methods and to improve the MSE and BER performances of the LS and MMSE channel estimate of the time dependent channel. The pilot placement problem is formulated as a combinatorial problem and the HBFOMCS optimization is employed to select optimal placement of the pilot tones. Simulation results show the superior performance of our novel algorithm for LS and MMSE methods over the other pilot tones design. The rest of this article is organized as follows. Section 2 introduces the channel estimation in MIMO-OFDM system. In Section 3, MIMO-OFDM system model is introduced. In section 4 , the pilot symbol based LS and MMSE channel estimation methods are concisely described while in Section 5, pilot design using HBFOMCS algorithm is proposed. Simulation results are provided in Section 6 and finally, Section 7 concludes our design.

\section{MIMO-OFDM SYSTEM MODEL}

A block diagram of a MIMO-OFDM system with $\mathrm{N}_{t}$ transmitter, $\mathrm{N}_{\mathrm{r}}$ receiver antennas and $\mathrm{R}$ subcarrier, is shown in Fig 1. The binary data symbols are first mapped according to the modulation type. After pilot symbols are inserted, $\mathrm{R}$ point Inverse Fast Fourier Transform (IFFT) converts the symbols into the time domain. Following the IFFT block at each transmitter antenna Cyclic Prefix (CP) is inserted to avoid Inter Symbol Interference (ISI). Then the symbols consisting of data and pilot symbols are transmitted from $i^{\text {th }}$ transmitter antenna.

After removing $\mathrm{CP}$ and taking the DFT at the $\mathrm{j}^{\text {th }}$ receiver antenna, the received $\mathrm{N} \times 1$ signal tones vector $Y_{j}(n)$ can be written as

$$
\begin{array}{r}
Y_{j}(n)=\sum_{i=1}^{N_{t}} X_{i}^{\operatorname{diag}}(n) F h_{i, j}+W_{j}(n) \\
i=1,2 \ldots \ldots N_{r} \quad j=1,2 \ldots \ldots N_{t}
\end{array}
$$

where $\mathrm{h}_{\mathrm{i}, \mathrm{j}}$ is $L$ length $L \times 1$ channel impulse response vector of belonging to $i^{\text {th }}$ transmitter antenna to $j{ }^{\text {th }}$ receiver antenna, $W_{j}(n)$ is the additive white Gaussian noise. $X_{i}(\mathrm{n})$ consist of $\mathrm{R} \times 1$ data vector $=S_{i}(\mathrm{n})$ and $\mathrm{R} \times 1$ pilot tones vector $=P_{i}(\mathrm{n})$ at time index $\mathrm{n}$.

$$
\mathrm{X}_{i}(n)=\mathrm{S}_{i}(n)+\mathrm{P}_{i}(n)
$$

$F$ is $(1 / \sqrt{N})$ times the $N \times N$ unitary DFT matrix

$$
F=\left[\begin{array}{ccc}
1 & 1 & 1 \\
1 & e^{-\frac{j 2 \pi}{N}} & e^{-\frac{j 2 \pi(N-1)}{N}} \\
\cdots & \cdots & \cdots \\
1 & e^{-\frac{j 2 \pi(N-1)}{N}} & e^{-\frac{j 2 \pi(N-1)(N-1)}{N}}
\end{array}\right]
$$

By assuming training over g consecutive OFDM symbols, we can write eq (1) as

$$
\begin{array}{r}
Y_{j}(n)=\sum_{i=0}^{N_{t}-1} S_{i}^{\operatorname{diag}}(n) F h_{i, j}+\sum_{i=0}^{N_{t}-1} P_{i}^{\operatorname{diag}}(n) F h_{i, j}+ \\
W_{j}(n)
\end{array}
$$

If we write equation (4) in simplified form

$$
Y_{j}=G h_{j}+A h_{h}+W_{j}
$$

where $\mathrm{G}=\left[P_{1}^{\text {diag }} F, \ldots, P_{N_{t}}^{\text {diag }} F\right]$ is $N \times N_{t} L$ sized matrix, $A=\left[S_{1}^{\text {diag }} F, \ldots ., S_{N_{t}}^{\text {diag }} F\right]$ is a $N \times N_{t} L . N_{t} L$ length channel impulse response of each antenna is given as

$$
h_{j}=\left[h_{j}, 1^{T} \ldots, h_{j}, N_{t}^{T}\right]^{T}
$$

where (.) ${ }^{\mathrm{T}}$ is transpose operation

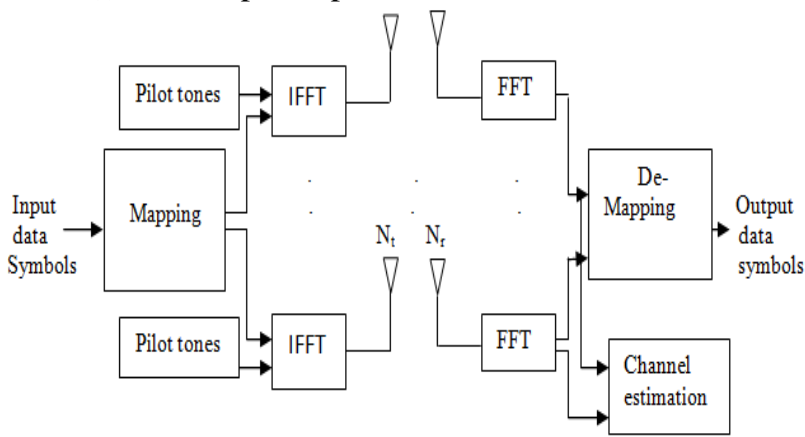

Fig. 1. MIMO-OFDM System

\section{CHANNEL ESTIMATION IN MIMO-OFDM SYSTEM}

Basically, methods of channel estimation can be classified according to four dimensions. From the view of estimation theory, there are Least Square (LS) Estimation and Minimum Mean Square Error (MMSE) estimation. According to the processing domain, estimation can be done in time domain and frequency domain. Due to the different pilot symbol arrangements, there are estimator with block type pilot (training based) and estimator with comb type pilot (pilot symbol aided modulation). With different estimation iteration, there are iterative methods and direct methods. Based on those assumptions such as perfect synchronization and block fading, we end up with a compact and simple signal model for both the single antenna OFDM and MIMO-OFDM systems.

In training based channel estimation algorithms, training symbols or pilot tones that are known to the receiver, are multiplexed along with the data stream for channel estimation. The idea behind these methods is to exploit knowledge of transmitted pilot symbols at the receiver to estimate the channel. For a block fading channel, where the channel is constant over a few OFDM symbols, the pilots are transmitted on all subcarriers in periodic intervals of OFDM blocks.

This type of pilot arrangement is called the block type arrangement. For a fast fading channel, where the channel changes between adjacent OFDM symbols, the pilots are transmitted at all times but with an even spacing on the subcarriers, representing a comb type pilot placement. The channel estimates from the pilot subcarriers are interpolated to estimate the channel at the data subcarriers. 


\section{A. LS Channel estimation}

The goal of the channel LS estimator is to minimize the square distance between the received signal and the original signal. The LS channel estimation method [14] finds the channel estimate $\hat{\mathrm{H}}$ in such a way that the following cost function is minimized

$$
\begin{aligned}
\mathrm{J}(\hat{\mathrm{H}}) & =\|\mathrm{Y}-\mathrm{X} \hat{\mathrm{H}}\|^{2} \\
& =(\mathrm{Y}-\mathrm{X} \hat{\mathrm{H}})^{\mathrm{H}}(\mathrm{Y}-\mathrm{X} \hat{\mathrm{H}}) \\
& =\mathrm{Y}^{\mathrm{H}} \mathrm{Y}-\mathrm{Y}^{\mathrm{H}} \mathrm{X} \hat{\mathrm{H}}-\hat{\mathrm{H}}^{\mathrm{H}} \mathrm{X}^{\mathrm{H}} \mathrm{Y}+\hat{\mathrm{H}}^{\mathrm{H}} \mathrm{X}^{\mathrm{H}} \mathrm{X}
\end{aligned}
$$

By setting the derivative of the function with respect to $\hat{H}$ to zero,

$\frac{\partial \mathrm{j}(\hat{\mathrm{H}})}{\partial \hat{\mathrm{H}}}=-2\left(\mathrm{X}^{\mathrm{H}} \mathrm{Y}\right)^{*}+2\left(\mathrm{X}^{\mathrm{H}} \mathrm{X} \hat{\mathrm{H}}\right)^{*}=0$

we have $\mathrm{X}^{\mathrm{H}} \mathrm{XH}=\mathrm{X}^{\mathrm{H}} \mathrm{Y}$, which gives the solution to the $\mathrm{LS}$ channel estimation as

$\hat{\mathrm{H}}_{\mathrm{LS}}=\left(\mathrm{X}^{\mathrm{H}} \mathrm{X}\right)^{-1} \mathrm{X}^{\mathrm{H}} \mathrm{Y}=\mathrm{X}^{-1} \mathrm{Y}$

Let us denote each component of the LS channel estimate $\hat{\mathrm{H}}_{\mathrm{LS}}$ by $\hat{\mathrm{H}}_{\mathrm{LS}}[\mathrm{k}], \mathrm{k}=0,1,2, \ldots, \mathrm{N}-1$. Since $\mathrm{X}$ is assumed to be diagonal due to the ICI-free condition, the LS channel estimate $\hat{H}_{L S}$ can be written for each subcarrier as

$\hat{\mathrm{H}}_{\mathrm{LS}}[\mathrm{k}]=\frac{\mathrm{Y}[\mathrm{k}]}{\mathrm{X}[\mathrm{k}]}, \mathrm{k}=0,1,2, \ldots, \mathrm{N}-1$

The Mean Square Error (MSE) of LS channel estimate is given as

$\mathrm{MSE}_{\mathrm{LS}}=\mathrm{E}\left\{\left(\mathrm{H}-\hat{\mathrm{H}}_{\mathrm{LS}}\right)^{\mathrm{H}}\left(\mathrm{H}-\hat{\mathrm{H}}_{\mathrm{LS}}\right)\right\}$

\section{B. MMSE Channel Estimation}

MMSE estimator has a better estimation accuracy compared to LS estimator in terms of MSE. However, the main short coming of the MMSE estimator is the higher computation complexity. With the increase of operation points, i.e., the number of subcarriers, its computational complexity will increase exponentially.

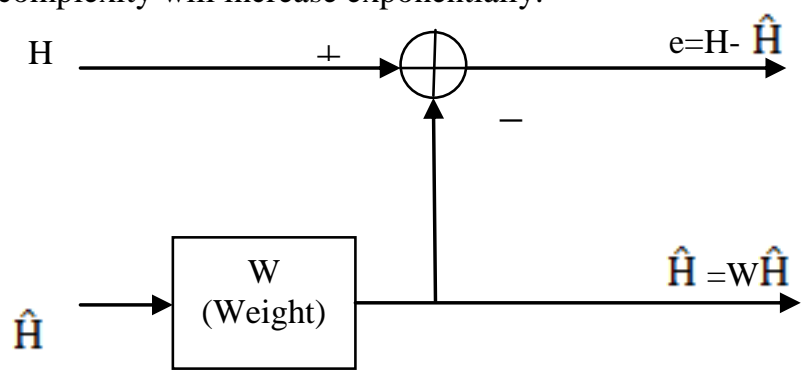

Fig. 2. MMSE Channel Estimation

Consider the LS solution in Equation (9), $\hat{\mathrm{H}}_{\mathrm{LS}}=\mathrm{X}^{-1} \mathrm{Y} \triangleq$ $\widetilde{\mathrm{H}}$. Using the weight matrix $\mathrm{W}$, define $\hat{\mathrm{H}}=\mathrm{W} \widetilde{\mathrm{H}}$, which corresponds to the MMSE estimate. MSE of the channel estimate $\hat{\mathrm{H}}$ is given as

$\mathrm{J}(\hat{\mathrm{H}})=\mathrm{E}\left\{\|\mathrm{e}\|^{2}\right\}=\mathrm{E}\left\{\|\mathrm{H}-\hat{\mathrm{H}}\|^{2}\right\}$

Then, the MMSE channel estimation method finds a better (linear) estimate in terms of $\mathrm{W}$ in such a way that the MSE in Equation 12 is minimized. MMSE channel estimation is as shown in Fig 2. The orthogonality principle states that the estimation error vector $\mathrm{e}=\mathrm{H}-\hat{\mathrm{H}}$ is orthogonal to $\hat{\mathrm{H}}$ such that

$$
\begin{aligned}
E\left\{e \widetilde{H}^{H}\right\} & =E\left\{(H-\hat{H}) \widetilde{H}^{H}\right\} \\
& =E\left\{(H-W \widetilde{H}) \widetilde{H}^{H}\right\} \\
& =E\left\{H \widetilde{H}^{H}\right\}-W E\left\{\widetilde{H} \widetilde{H}^{H}\right\} \\
& =R_{H} \widetilde{H}^{-1}{ }_{\widetilde{H} \widetilde{H}}=0
\end{aligned}
$$

where $R_{A B}$ is the cross-correlation matrix of $N \times N$ matrices $\mathrm{A}$ and $\mathrm{B}$ (i.e., $\mathrm{R}_{\mathrm{AB}}=\mathrm{E}\left[\mathrm{AB}^{\mathrm{H}}\right]$, and $\widetilde{\mathrm{H}}$ is the $\mathrm{LS}$ channel estimate given as

$\widetilde{\mathrm{H}}=\mathrm{X}^{-1} \mathrm{Y}=\mathrm{H}+\mathrm{X}^{-1} \mathrm{Z}$

Solving Equation (13) for W yields

$\mathrm{W}=\mathrm{R}_{\mathrm{H} \widetilde{\mathrm{H}}} \mathrm{R}^{-1} \widetilde{\mathrm{H}} \widetilde{\mathrm{H}}$

$\mathrm{R}_{\mathrm{H} \widetilde{\mathrm{H}}}$ is the cross-correlation matrix between the true channel vector and temporary channel estimate vector in the frequency domain.Using Equation (16), the MMSE channel estimate follows as

$$
\begin{aligned}
& \text { MSE }_{M M S E}=E\left\{\left(H-\widehat{H}_{M M S E}\right)^{H}\left(H-\widehat{H}_{M M S E}\right)\right\} \\
& \text { where } \widehat{H}_{M M S E}=W \widetilde{H}=R_{H \widetilde{H}} R^{-1} \widetilde{H} \widetilde{H}^{\widetilde{H}}
\end{aligned}
$$

\section{PILOT TONES OPTIMIZATION IN LS AND MMSE CHANNEL ESTIMATION METHOD}

For LS and MMSE channel estimation method, an exhaustive search of all possible pilot patterns for the best pilot pattern with the minimum objective is performed. A huge search space with $\mathrm{C}_{128}^{16} \approx 2.26041 \times 10^{28}$ searches is possible for 128 subcarriers and 16 pilot symbols. For this combinatorial optimization problem, a scheme using stochastic optimization algorithm is proposed that is iterative in nature and converge to the global optimum in probability. The scheme is based on the Hybrid of Bacterial Foraging and Modified cuckoo Search (HBFOMCS) optimization algorithm. HBFOMCS algorithm is evaluated in MIMO-OFDM system environment due to obtain significant MSE and BER performances.

\section{A. Hybrid of Bacterial Foraging and Modified Cuckoo Search (HBFOMCS) Algorithm}

Optimization has been a popular research topic for decades. Especially, dynamic optimization problems have objective functions changing over time, which causes changes in the position of optima as well as the characteristics of the search space. This leads to the fact that existing optima may disappear, while new optima may appear. Optimization under the dynamic environments is a challenging task that attracts great attention. During recent years, biology inspired soft computing methods have been widely used in different optimization problem solving cases. The BFO [15] and MCS [16] algorithms are emerging approaches mimicking the foraging behavior of Bacteria and Cuckoos. However, these powerful optimization methods have their inherent shortcomings and limitations. It is known that, fusion of the computational intelligence methodologies can usually 
provide superior performances over employing them individually. Therefore, a hybrid algorithm based on the BFO and MCS (HBFOMCS) is proposed to cope with complex optimization problem in channel estimation.

The novel HBFOMCS algorithm for LS and MMSE Channel estimation method is designed by considering the limitations of pilot tones design for MIMO-OFDM system. The optimization of placement of pilot tones using HBFOMCS algorithm for MIMO-OFDM environment is evaluated by considering the following conditions

- Placement of pilot tones at the start and end of the subcarriers of OFDM are restricted.

- Pilot tones are optimized by considering all the subcarrier locations to produce better MSE values.

- Optimized pilot tones should not occupy the same position in the available subcarrier locations.

- Spacing between the pilot tones is calculated by considering the effect of Doppler frequencies, which is also taken into consideration for optimization.

HBFOMCS algorithm [17],[18] is implemented to determine the optimal pilot tones location with reduced computational complexity in order to improve the Mean Square Error (MSE) and Bit Error Rate (BER) channel estimation performances. The procedure for the proposed algorithm to solve pilot tones optimization problem in CR environment is as follows.

\section{Step1) LUs, Pilot locations and HBFOMCS parameters specification}

Specify the number of subcarriers (M), number of pilots $\left(\mathrm{cp}_{\mathrm{k}}\right)$ to determine the number of possible pilot location combinations available for optimization and the number of LUs. Specify the control parameters of HBFOMCS algorithm such as initial population $(\mathrm{N})$, number of Generations (G), Maximum Swim $\left(\mathrm{M}_{\mathrm{s}}\right)$, Maximum Tumble $\left(\mathrm{M}_{\mathrm{t}}\right)$ and Discover probability $\left(\mathrm{P}_{\mathrm{d}}\right)$.

Step 2) Initialization of population with random solutions

The initial population or solutions are generated randomly in the multidimensional solution of RUs location space, where the population represents the available RUs locations pilot combinations. Depending upon the presence of LUs, $\mathrm{N}$ pilot location combinations are initially chosen from the available RUs locations pilot combinations. In their initial positions $j$, the fitness function i.e.MSE (i) values are calculated.

$$
\operatorname{MSE}(i)=\operatorname{MSE}_{L S}(i)=\operatorname{MSE}_{M M S E}(i)
$$

$P_{d}$ is initialized with a value between 0 and 1.The values of $G, M_{s}$ and $M_{t}$ are also initialized.

\section{Step 3) Swim and Tumble}

a. Swim: Calculate MSE, $\operatorname{MSE}^{\text {new }}(\mathrm{i})$ at new position $\mathrm{j}+\mathrm{K}$, $\mathrm{K}=1,2, \ldots, \mathrm{N}_{\mathrm{s}}$. Check $\mathrm{MSE}^{\text {new }}(\mathrm{i})<\quad \mathrm{MSE}(\mathrm{i}) \quad$ or $\operatorname{MSE}^{\text {new }}(\mathrm{i})=\mathrm{MSE}(\mathrm{i})$. Continue swim up to $\mathrm{N}_{\mathrm{s}}$ swim steps.

b. Tumble: If $\mathrm{MSE}^{\text {new }}(\mathrm{i})>\mathrm{MSE}(\mathrm{i})$ within $\mathrm{N}_{\mathrm{s}}$ swim steps tumble is initiated. Here tumble denotes one unit walk in random direction and gets new pilot location combinations and it continues upto initialized $\mathrm{M}_{\mathrm{t}}$ value.
On the completion of swim and tumble, the new pilot location positions are ensured that they are placed only in RUs location.

Step 4) Discover Probability $\left(P_{d}\right)$

- $\mathrm{N}$ optimal pilot location combinations are grouped together by sharing their fitness i.e. they are arranged in descending order based on their MSE values.

- Arranged $\mathrm{N}$ optimal pilot location combinations are grouped using the split position $. \mathrm{S}=\left(\mathrm{Ps}^{*} \mathrm{~N}\right)$, where $\mathrm{P}_{\mathrm{s}}$ $=1-\mathrm{P}_{\mathrm{d}}, \mathrm{P}_{\mathrm{s}}-$ Split function.

- The pilot location combinations are grouped as

\section{Step 5) Reproduction using Levy flight}

- Levy flight is used to generate the new pilot location combinations using Max Levy step `size A.

- Levy flight elects new pilot location combinations with A $\varepsilon[1,3]$. The process is repeated until the bottom pilot location combinations are replaced by new pilot location combinations.

- The generation of top pilot location combinations is slightly different from the bottom combinations. Here Levy flight elects new pilot location combinations with A $\epsilon[1,3]$.The MSE value, $\operatorname{MSE}^{\text {new }}(i)$ of the new pilot location combinations will be compared with the MSE(i) of all the combinations available in the top and bottom position of combinations. If $\mathrm{MSE}^{\text {new }}(\mathrm{i})$ is less than or equal to MSE(i) then MSE(i) is replaced with $\operatorname{MSE}^{\text {new }}(i)$ else MSE(i) is not replaced.

- Once all the top and bottom position pilot location combinations are replaced by new pilot location combinations via MCS-Levy flight, it contributes a generation. On the completion of Levy flight, the new pilot location positions are ensured that they are placed only in RUs location.

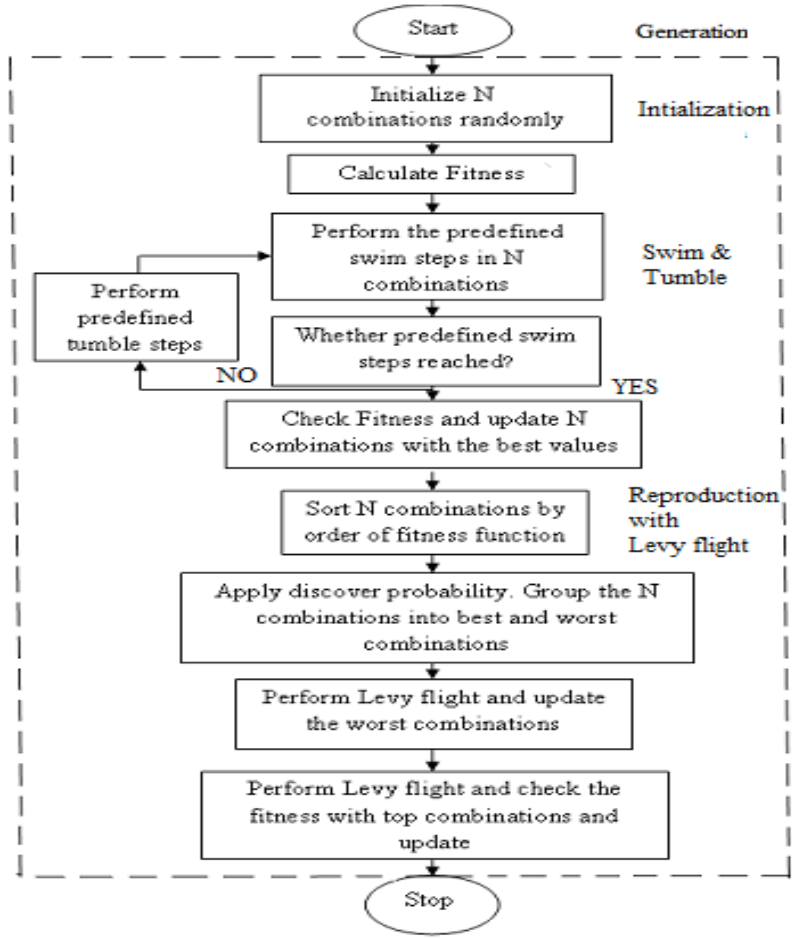

Fig. 3. HBFOMCS Algorithm Flow diagram 
- The pilot location combinations with the best MSE values, MSE (i) are placed in the next generation. Step 2 to Step 5 is repeated until $\mathrm{G}$ generations are completed. The optimum pilot location combinations producing best MSE value is obtained once $G$ generations are completed. The Computational Complexity of the proposed HBFOMCS based pilot symbol placement is calculated as $\mathrm{N}^{*} \mathrm{G}$, where $\mathrm{N}$ is the initial population or pilot location combinations and $\mathrm{G}$ is the number of generations. The flow diagram of the proposed HBFOMCS algorithm is as shown in Fig 3.

\section{SIMULATION RESULTS}

The LS and MMSE channel estimation based MIMOOFDM system using HBFOMCS approach for optimization of pilot tones placement with reduced computational complexity is validated, in the MATLAB environment. The applicability and validity of the algorithms for MSE and BER performances have been tested for various subcarriers and Doppler frequency values.

\section{A. Setting of HBFOMCS Parameters}

The various control parameters of HBFOMCS algorithm such as the initial population, the number of generation, maximum swim and tumble and the discover probability value are to be set before its implementation. Setting of these algorithm parameters optimally can yield better solution for channel estimation with lesser computational complexity. In this work, the simulation parameters of HBFOMCS algorithm utilized for different subcarriers and pilots are illustrated in the Table I

TABLE I. SIMULATION PARAMETERS OF HBFOMCS ALGORITHM

\begin{tabular}{|l|l|l|}
\hline \multicolumn{1}{|c|}{ Simulation Parameters } & $\begin{array}{c}\mathbf{1 2 8} \\
\text { Subcarrier }\end{array}$ & $\begin{array}{c}\mathbf{6 4} \\
\text { Subcarrier }\end{array}$ \\
\hline Initial population(N) & 40 & 20 \\
\hline Number of Generations $(\mathrm{G})$ & 50 & 50 \\
\hline Max Swim $\left(\mathrm{N}_{\mathrm{s}}\right)$ & 3 & 3 \\
\hline Max Tumble $\left(\mathrm{N}_{\mathrm{t}}\right)$ & 2 & 2 \\
\hline Discover Probability $\left(\mathrm{P}_{\mathrm{D}}\right)$ & 0.4 & 0.4 \\
\hline
\end{tabular}

\section{B. Setting of MIMO-OFDM System Parameters}

The MIMO-OFDM system is designed and simulated by utilizing the following parameters as shown in Table II. MIMO-OFDM system is simulated using QPSK modulation with 64 and 128 subcarriers. The MSE and BER values are computed by considering two transmitting and receiving antennas for Doppler frequency values of $f_{d}$ $=5 \mathrm{~Hz}$ and $40 \mathrm{~Hz}$ respectively.

TABLE II. SIMULATION PARAMETERS OF MIMO-OFDM SYSTEM

\begin{tabular}{|l|c|c|}
\hline \multicolumn{1}{|c|}{ Parameter } & Value & Value \\
\hline Number of subcarrier & 128 & 64 \\
\hline FFT size & 128 & 64 \\
\hline Number of pilot symbol & 16 & 8 \\
\hline Modulation type & QPSK & QPSK \\
\hline OFDM symbol duration $(\tau \mathrm{s})$ & $1.13 \mathrm{~ms}$ & $565 \mu \mathrm{s}$ \\
\hline
\end{tabular}

The MSE and BER values are computed by considering two transmitting and receiving antennas for Doppler frequency values of $f_{d}=5 \mathrm{~Hz}$ and $40 \mathrm{~Hz}$ respectively.In simulations the channel estimation performances of various pilot tones placement are evaluated such as

$\checkmark$ Random pilot tones placement.

$\checkmark$ Equispaced orthogonal pilot tones placement

$\checkmark$ BFO optimized LS based pilot tones placement

$\checkmark$ MCS optimized LS based pilot tones placement

$\checkmark$ HBFOMCS optimized LS based pilot tones placement.

$\checkmark$ HBFOMCS optimized MMSE based pilot tones placement.

In Fig 4, MSE of different pilot tones for 128 subcarriers over channels with Doppler frequency shift of $f_{d}=5 \mathrm{~Hz}$ are shown. The proposed HBFOMCS based MMSE channel estimation (HBFOMCS-MMSE) gives improved MSE values especially at low SNR compared with HBFOMCS based LS channel estimation (HBFOMCS-LS)and other placement techniques.

From Figure 3., it is observed that the random placement requires $27 \mathrm{~dB}$ of $\mathrm{SNR}$, orthogonal placement requires 25 $\mathrm{dB}$ of SNR, HBFOMCS-LS based placement requires 14 $\mathrm{dB}$ of SNR, whereas HBFOMCS-MMSE based placement requires only $12 \mathrm{~dB}$ of SNR to have MSE $=10^{-3}$. At $0 \mathrm{~dB}$ SNR value, the MSE difference between orthogonal and HBFOMCS-LS based placement is $10^{-1}$, this difference between HBFOMCS-MMSE and HBFOMCS-LS placement is about $10^{-1}$.

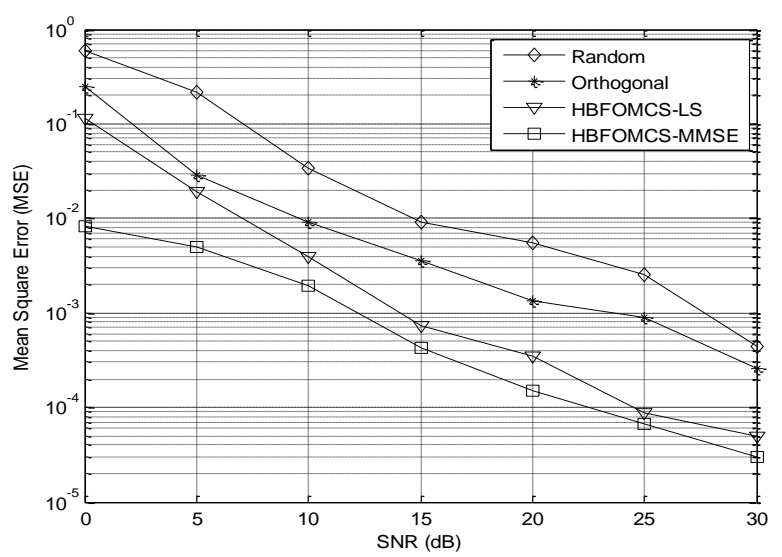

Fig. 4. MSE versus SNR for various pilot tones with 128 subcarriers $\quad\left(f_{d}=5 \mathrm{~Hz}\right)$

The MSE performances of HBFOMCS-MMSE over the channel with increased Doppler shift are simulated for $f_{d}=40 \mathrm{~Hz}$ as shown in Fig 5. From Fig 4 and Fig 5, it is observed that degradation of MSE performance for HBFOMCS-MMSE of about $0.4 * 10^{-1}$ at $30 \mathrm{~dB}$ SNR value occurs with the increase in Doppler shift value.

Also to show the effect of number of subcarrier on system performance, MSE of the systems which have 64 subcarriers with $f_{d}=5 \mathrm{~Hz}$ and $40 \mathrm{~Hz}$ are simulated in Fig 6 and Fig 7. According to these figures, system performance is decreased with the reduction of the subcarrier number. For instance, considering Fig 6 and Fig 7, at $30 \mathrm{~dB}$ SNR value the MSE difference of optimized pilot tones using HBFOMCS-MMSE algorithm is approximately $0.8 * 10^{-1}$. 


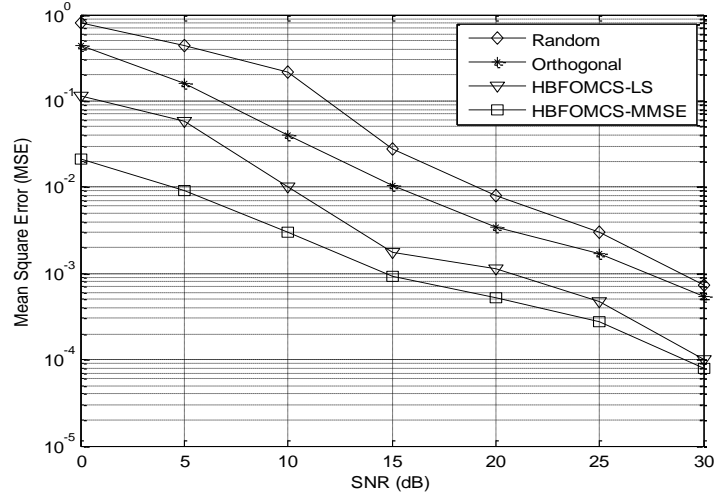

Fig. 5. MSE versus SNR for various pilot tones with 128 subcarriers $\quad\left(f_{d}=40 \mathrm{~Hz}\right)$

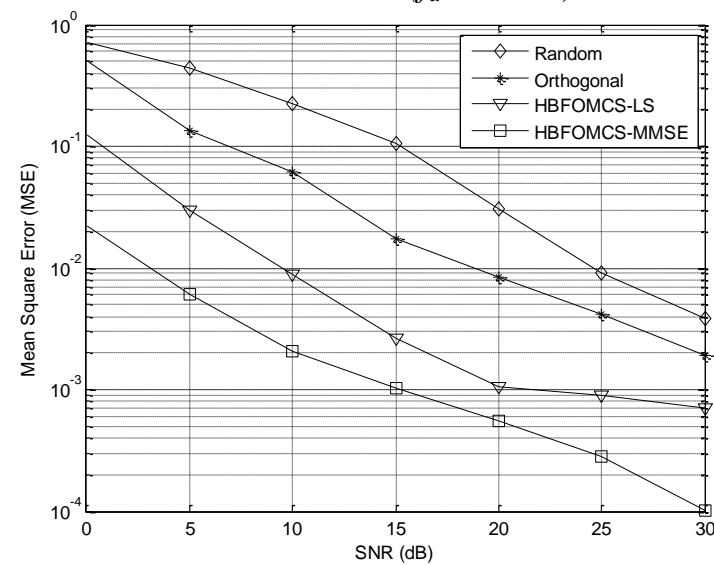

Fig. 6. MSE versus SNR for various pilot tones with 64 subcarriers $\quad\left(f_{d}=5 \mathrm{~Hz}\right)$

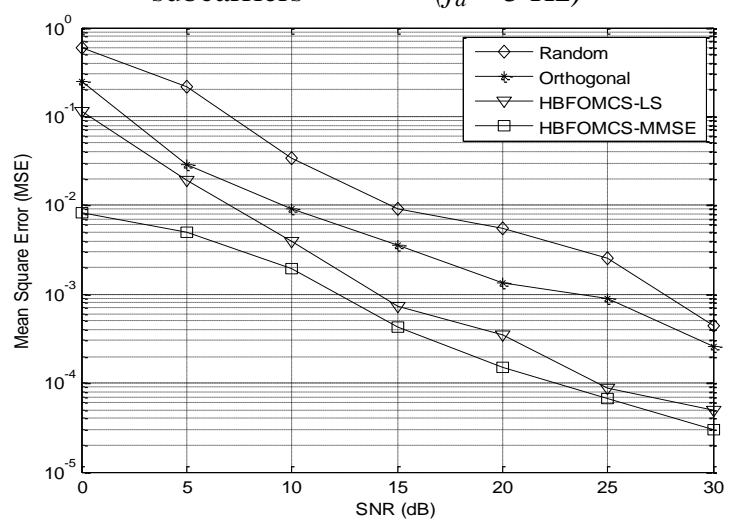

Fig. 7. MSE versus SNR for various pilot tones with 64 subcarriers $\quad\left(f_{d}=40 \mathrm{~Hz}\right)$

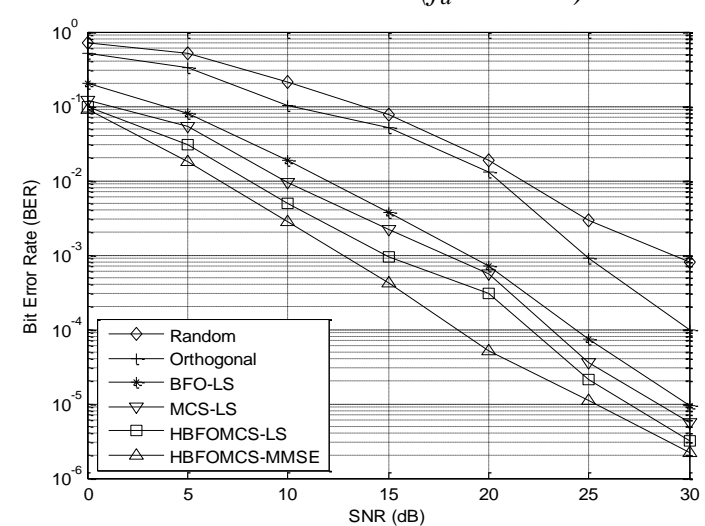

Fig. 8. BER versus SNR for various pilot tones with128 subcarriers

$\left(f_{d}=5 \mathrm{~Hz}\right)$
Fig 8 illustrates the Bit Error Rate (BER) performances of the pilot tones design for Doppler shift $f_{d}=5 \mathrm{~Hz}$. As it can be seen from Fig 8, HBFOMCS-MMSE based pilot tones design performs better than the other pilot tones design techniques.

HBFOMCS-MMSE and HBFOMCS-LS based placement techniques shows about $12 \mathrm{~dB}$ and $10 \mathrm{~dB}$ SNR advantage relative to the orthogonal placement at the BER of $10^{-3}$. HBFOMCS-MMSE advantage over HBFOMCS-LS placement is about $2 \mathrm{~dB}$ at the BER of $10^{-3}$. HBFOMCSMMSE also performs better than BFO and MCS based placement technique of about $5 \mathrm{~dB}$ and $6 \mathrm{~dB}$ SNR value at the BER near $10^{-3}$. At $10^{-3}$ BER, HBFOMCS-MMSE achieves a SNR gain of about $5 \mathrm{~dB}$ over ABC [Seyman \& Taspinar 2013], $7 \mathrm{~dB}$ over PSO [Seyman \& Taspinar 2011] and DE [Seyman \& Taspinar 2012] assisted pilot tones strategy.

Besides, the BER values of the pilot tones placement strategies for Doppler shift $f_{d}=40 \mathrm{~Hz}$ are shown in Fig 9 . Considering Fig 9, there is $15 \mathrm{~dB}$ SNR difference between HBFOMCS-MMSE and orthogonal placement of pilot symbol at BER of $10^{-3}$.Also, there is $3 \mathrm{~dB}$ SNR difference between HBFOMCS-MMSE and HBFOMCS-LS placement of pilot symbol at BER of $10^{-3}$. Furthermore to have $\mathrm{BER}=10^{-3}$, PSO based placement needs $24 \mathrm{~dB}, \mathrm{DE}$ based placement needs $23 \mathrm{~dB}$, $\mathrm{ABC}$ based placement needs $22 \mathrm{~dB}$ and HBFOMCS-MMSE placement needs only $13 \mathrm{~dB}$ of SNR value.

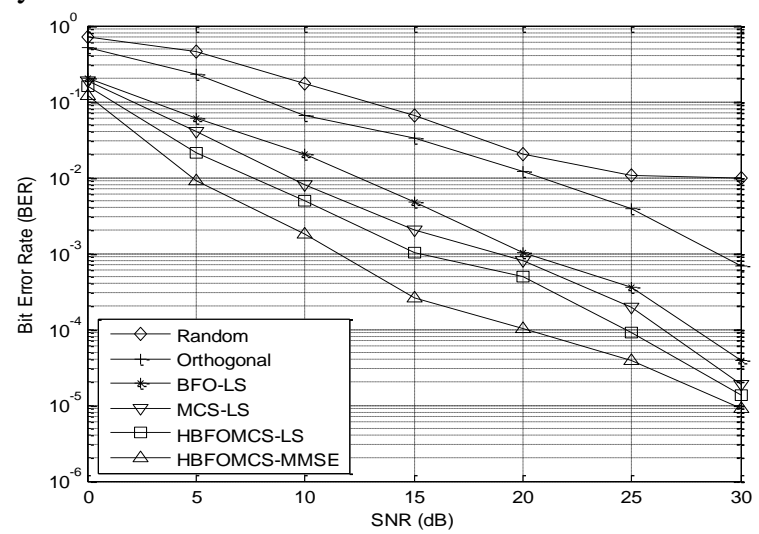

Fig. 9. BER versus SNR for various pilot tones with128

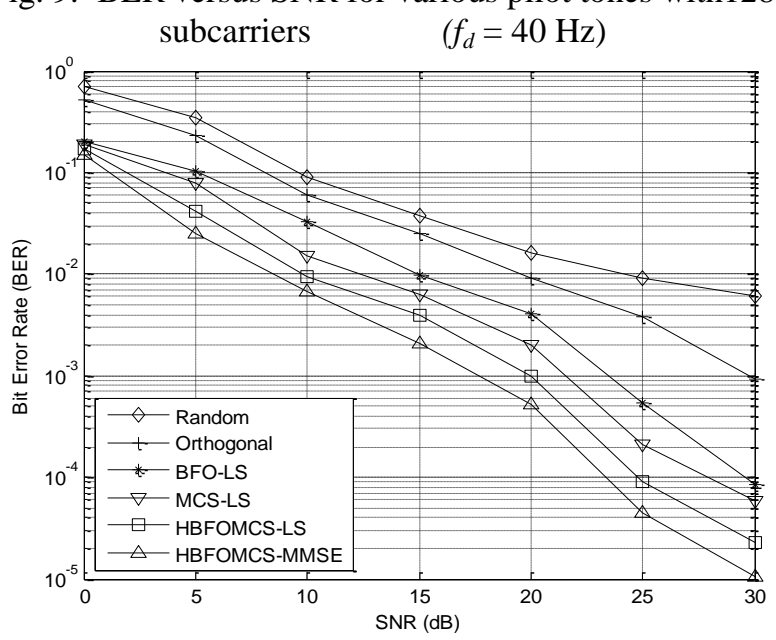

Fig. 10. BER versus SNR for various pilot tones with 64 subcarriers $\quad\left(f_{d}=5 \mathrm{~Hz}\right)$ 
Also to show the effect of number of subcarrier on system performance, BER of the systems which have 64 subcarriers with $f_{d}=5 \mathrm{~Hz}$ and $40 \mathrm{~Hz}$ are simulated in Fig 10 and Fig 11. According to these figures, system performance is decreased with the reduction of the subcarrier number. For instance, considering Fig 10 and 11 , at $30 \mathrm{~dB}$ SNR value the BER difference of HBFOMCS-MMSE optimized pilot tones is approximately $10^{-1}$

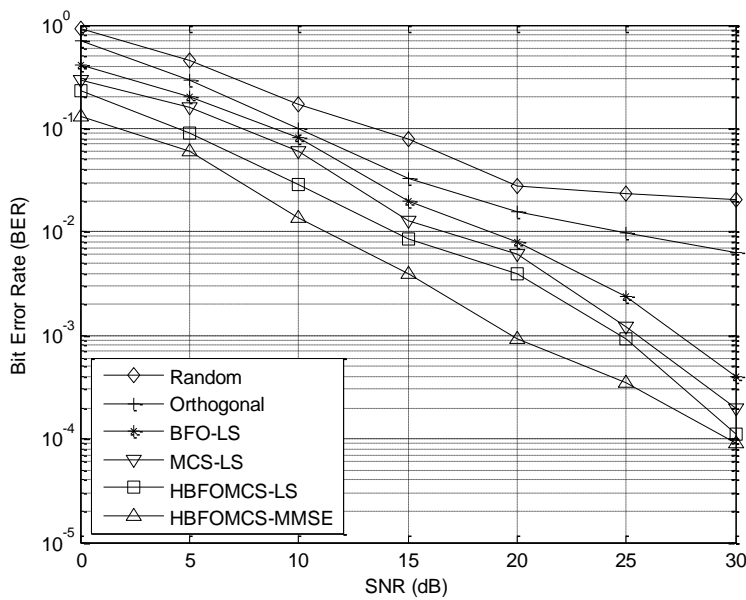

Fig. 11. BER versus SNR for various pilot tones with 64 subcarriers $\quad\left(f_{d}=40 \mathrm{~Hz}\right)$

In the simulation graphs from Fig 4 to Figure 11, 64 and 128 subcarriers were used along with 1000 data symbols. This simulation parameters result in better MSE and BER performances when compared with the existing optimization algorithms such as ABC[Seyman \& Taspinar 2013], PSO [Seyman \& Taspinar 2011] and DE [Seyman \& Taspinar 2012] assisted pilot tones strategy. The simulations can be performed for larger subcarriers and data symbols, but as the configuration of the simulating systems is not so high enough it may be time consuming.

\section{CONCLUSION}

In this paper, we have proposed an efficient pilot tones design method based on the hybrid BFOMCS algorithm to optimize the placement of pilot tones that are used in the LS and MMSE channel estimation of MIMO-OFDM systems. According to the simulation results, our proposal performs better than random, eqispaced orthogonal based placement strategies for various Doppler shifts. Furthermore, this method has the computational complexity advantage over the orthogonal placement of pilot symbols. Hence it is concluded that the HBFOMCS algorithm is an alternative solution to achieve both highperformance efficiency and low-computational complexity in designing pilot symbols for LS and MMSE channel estimation of MIMO-OFDM system.

\section{REFERENCES}

[1] Lee, KF \& Willians, DB 2000, A Space-Time Coded Transmit Diversity Technique For Frequency Selective Fading Channels ${ }^{e c}$, Proceedings of IEEE Sensor Array and Multichannel Signal Processing Workshop, pp. 149-152.

2] Kansal, L, Kansal, A \& Singh, K 2011, „Performance Analysis of MIMO-OFDM System Using QOSTBC Code Structure for MQAM”, Canadian Journal on Signal Processing, vol. 2, no. 2.
[3] Bahai, AR, Saltzberg, BR 1999, „Multi-Carrier Digital Communications -Theory and Applications of OFDM"e, Kluwer Academic/Plenum.

4] Omri, A \& Bouallegue, R 2011, „New Transmission Scheme for MIMO-OFDM System", International Journal of Next-Generation Networks, vol. 3, no. 1 .

[5] $\mathrm{Hu}, \mathrm{D}$ and $\mathrm{He}, \mathrm{L}$ 2010, 'Pilot design for channel estimation in OFDM-based cognitive radio systems', Proc. IEEE Global Telecommunications Conf. (GLOBECOM (2010), pp. 1-5

[6] Petropulu, A, Zhang, R \& Lin, R 2004, 'Blind OFDM channel estimation through simple linear pre-coding', IEEE Transactions on Wireless Communications, vol. 3, no. 2, pp. 647-655.

[7] Panah, AY, Vaughan, RG \& Heath, RW 2009, „Optimizing pilot locations using feedback in OFDM systems ${ }^{\text {ee }}$, IEEE Transactions onVehicular Technology, vol. 58, pp. 2803-2814.

[8] Barhumi, I, Leus, G \& Moonen, M 2003, „Optimal Training Design for MIMO OFDM Systems in Mobile Wireless Channelse ${ }^{\text {ee }}$ IEEE Transactions on Signal Processing, vol. 51, no. 6, pp. 1615-1624.

[9] Coleri, S, Ergen, M, Puri, A \& Bahai, A 2002, 'Channel estimation techniques based on pilot arrangement in OFDM systems", IEEE Transactions on Broadcasting, vol. 48, pp. 223-229.

[10] $\mathrm{Hu}, \mathrm{D}, \mathrm{He}, \mathrm{L}$ and Wang,X 2011, ' An efficient pilot design method for OFDM-based cognitive radio systems', IEEE Transaction on Wireless Communication, vol.10, no 4,pp. 1252-1259.

[11] Muhammet Nuri Seyman \& Necmi Taspınar 2013, 'Pilot tones optimization Using Artificial Bee Colony algorithm for MIMOOFDM Systems', Wireless Personal Communication, vol. 71, pp. 151-163.

[12] Muhammet Nuri Seyman \& Necmi Taspinar 2012, 'Optimization of pilot tones using differential evolution algorithm in MIMO-OFDM systems', Turkish Journal of Electrical Eng \& Comp Science, vol. 20, no. 1 , pp. $15-23$

[13] Muhammet Nuri Seyman \& Necmi Taspınar 2011, 'Particle swarm optimization for pilot tones design in MIMO-OFDM systems', EURASIP Journal on Advances in Signal Processing, vol. 1, no. 10.

[14] Yong Soo Cho, Jaekwon Kim, Won Young Yang \& Chung Kang, G 2010, „MIMO-OFDM Wireless Communications with MATLAB $^{e}$, Wiley Publications.

[15] R.Manjith, M.Suganthi,A suboptimal PTS algorithm based on bacterial foraging optimization for PAPR reduction in MIMOOFDM system, Journal of theoretical and applied information technology, vol 57, no 2, pp 261-268,2013.

[16] R.Manjith, M.Suganthi ,Peak to average power ratio reduction using modified cuckoo search algorithm in MIMO-OFDM system, Australian journal of basic and applied sciences, November, pp 3242,2013.

[17] R.Manjith, M.Suganthi ,Peak to average power ratio reduction using a Hybrid of Bacterial Foraging and modified cuckoo search algorithm in MIMO-OFDM system, Research Journal of Applied Sciences, Engineering and Technology 7(21): 4423-4433, 2014

[18] R.Manjith, M.Suganthi , A Hybrid of Bacterial Foraging and Modified Cuckoo Search optimization for pilot symbol design in MIMO-OFDM Systems, Research Journal of Applied Sciences, Engineering and Technology 8(6): 726-735, 2014 